\title{
Correction to: A Selective Review of Negative Control Methods in Epidemiology
}

\author{
$\mathrm{Xu} \mathrm{Shi}^{1}$ (D) Wang Miao ${ }^{2} \cdot$ Eric Tchetgen Tchetgen $^{3}$ \\ Published online: 8 May 2021 \\ (C) Springer Nature Switzerland AG 2021
}

\section{Correction to: Current Epidemiology Reports (2020) 7:190-202 https://doi.org/10.1007/s40471-020-00243-4}

The original article unfortunately needs corrections.

(1) Page 194 Assumption 5: please change from " $W \perp f \perp U$ $\mid X$ and $Z \perp f \perp U \mid A, X$ ” to " $W \not \perp U \mid X$ and $Z \not \perp U \mid A, X$ "

(2) Page 195 Table 2: could you make sure that all reference numbers greater than 50 are added by one in this table (during the production process last time, a newly published paper was added and cited as [50], but in Table 2 the subsequent reference numbers did not get to be shifted by one). Specifically,

(a) Line 3 of Table 2: change from "[60,61]" to "[61,62]"

(b) Line 5 of Table 2: change from "[62]" to "[63]"

(c) Line 7 of Table 2: change from "[63,64]" to "[64,65]"

(d) Line 8 of Table 2: change from "[ $[13,65]$ " to " $[13,66]$ "

(e) Line 9 of Table 2: change from "[66]" to "[67]"

(f) Line 10 of Table 2: change from "[67-69]" to "[68-70]"

(3) Page 196 Assumption 7: please change from " $W \nVdash Z \mid A$ $=a, X$ " to " $W \not \not Z \mid A=a, X$ "

The online version of the original article can be found at https://doi.org/ $10.1007 / \mathrm{s} 40471-020-00243-4$

Xu Shi

shixu@umich.edu

1 Department of Biostatistics, University of Michigan, Ann Arbor, MI, USA

2 Department of Probability and Statistics, Peking University, Beijing, China

3 Statistics Department, The Wharton School, University of Pennsylvania, Philadelphia, PA, USA
(4) Page 196 line 16 in the right column of text (the line above the Rationale section): please add to the end of the sentence: "is not equal to zero for all $a, x$."

(5) Page 197 line 15 from the bottom in the left column of text (4th line below Eq. (7)): please change from " $\beta_{Y U}$ $\beta_{Y A}$ " to " $\beta_{Y U} \beta_{U A}$ "

(6) Page 197 line 2 from the bottom in the left column of text (17th line below Eq. (7)): please change from " $\beta_{Y U} \beta_{Y A}$ " to " $\beta_{Y U} \beta_{U A}$ "

(7) Page 197 line 26 (line 7 of second paragraph) in the right column of text: please change from " $\beta_{Y U} \beta_{Y A}$ " to " $\beta_{Y U} \beta_{U A}$ "

(8) Page 197 line 9 from the bottom in the right column of text: please change from "Because $Z-Y(Z-W)$ association is a product of $Z-Y$ and $Z-Y(Z-Y)$ associations" to "Because $Z-Y$ (or $Z-W$ ) association is a product of $Z-U$ and $U-Y$ (or $U-W$ ) associations"

(9) Page 198 4th line above Eq. (10) in the left column of text: please change from " $\widetilde{U}_{W}=\frac{W-\beta_{0}}{\beta_{W U}}$ " to " $\widetilde{U}_{W}=\frac{W-\beta_{W 0}}{\beta_{W U}}$ "

(10) Page 198 3rd line above Eq. (12) in the left column of text: please change the term between the two equal signs from " $E u\{E[Y \mid A=a, U]\}$ " to " $E_{U}\{E[Y \mid A=a, U]\}$ " (that is, change "u" after the first " $E$ " to subscript).

(11) Page 198 line 4 in the right column of text: please change from "Stage I: regress $\mathrm{W}$ on $\mathrm{A}$ and $\mathrm{Z}$ and obtain" to "Stage I: regress W on A and Z to obtain"

(12) Page 198 line 5 in the right column of text: could you please start a new paragraph starting from "Stage II"; then another new paragraph starting from "The twostage least squares approach given above provides ...."

(13) Page 199 line 3 in the left column of text: please add indent before "Violation 1" (like what was done for "Violation 2", "Violation 3", and "Violation 4")

(14) Page 199 line 9 in the right column of text (last line of "Violation 4" paragraph): please change from "This violates Assumption 2" to "This violates Assumption 4" 
Table 3 Examples of graphs for $Z, A, U$ relationships and for $W, Y, U$ relationships. The two pieces of graphs can be combined in to a directed acyclic graph that encodes the negative control assumptions. Grey colored graphs are invalid because of violation of key assumptions

\begin{tabular}{|c|c|c|c|}
\hline & \multicolumn{5}{|c|}{ Examples of graphs tor $Z, A, U$ relationships } \\
\hline No arrow between & Instrumental variable (IV) & Violate Assumptions 5 and 7 & Violate Assumptions 5 and 7 \\
$U$ and $Z$ (may violate & & \\
Assumptions 5 and 7) & & \\
\hline
\end{tabular}

(15) Page 193 line 9 from the bottom in the right column of text: please change from " $[32,33,49,51]$ specify future measures of ..." to past tense "[32,33,49,51] specified future measures of ..."

(16) Page 199 Table 3: please substitute the current table with the table attached in the email

(17) Page 200 References:

(a) Ref 1: please change author from "John PA Ioannidis" to "Ioannidis JPA"

(b) Ref 10: please change author from "Lan Liu and Eric Tchetgen Tchetgen" to "Liu L, Tchetgen Tchetgen EJ"

(c) Ref 11: in the title, please change from "Stationarity" to "Stationarity"

(d) Ref 15: in the author list, please change from "TP Speed" to "Speed TP"

(e) Ref 18: in the journal name, please change from "Pharmacoepidemi- ology" to "Pharma coepidemiology"

(f) Ref 33: in the title, please change from "par- tial" to "partial"

(g) Ref 44: in the author list, please change from "et al" to "Lawlor DA" (then we would have the full author list and do not need et al) (h) Ref 47: in the title, please change from "off- spring" to "offspring"

(i) Ref 54: in the title, please change from "com- paring" to "comparing"

(j) Ref 58: please change author from "Bradford Hill A" to "Hill BA"

(k) Ref 59: please change author from "Cuyler Hammond E" to "Hammond EC"

(1) Ref 61: in the title, please change from "stud- ies" to "studies"

(m) Ref 67: please change author from "Tchetgen EJ" to "Tchetgen Tchetgen EJ"

(n) Ref 70: in the title, please change from "adjustment" to "adjustment"

(o) Ref 71: in the title, please change from "nonparametric" to "nonparametric"

The original article has been corrected.

Publisher's Note Springer Nature remains neutral with regard to jurisdictional claims in published maps and institutional affiliations. 